\title{
Treatment of plaque psoriasis with an ointment formulation of the Janus kinase inhibitor, tofacitinib: a Phase $2 b$ randomized clinical trial
}

Kim A. Papp ${ }^{1}$, Robert Bissonnette ${ }^{2}$, Melinda Gooderham³ ${ }^{3}$ Steven R. Feldman ${ }^{4}$, Lars Iversen ${ }^{5}$, Jennifer Soung ${ }^{6}$, Zoe Draelos ${ }^{7}$, Carla Mamolo ${ }^{8}$, Vivek Purohit ${ }^{8}$, Cunshan Wang ${ }^{8}$ and William C. Ports ${ }^{8^{*}}$

\begin{abstract}
Background: Most psoriasis patients have mild to moderate disease, commonly treated topically. Current topical agents have limited efficacy and undesirable side effects associated with long-term use. Tofacitinib is a small molecule Janus kinase inhibitor investigated for the topical treatment of psoriasis.

Methods: This was a 12-week, randomized, double-blind, parallel-group, vehicle-controlled Phase 2b study of tofacitinib ointment (2\% and $1 \%$ ) applied once (QD) or twice (BID) daily in adults with mild to moderate plaque psoriasis. Primary endpoint: proportion of patients with Calculated Physician's Global Assessment (PGA-C) clear or almost clear and $\geq 2$ grade improvement from baseline at Weeks 8 and 12. Secondary endpoints: proportion of patients with PGA-C clear or almost clear; proportion achieving Psoriasis Area and Severity Index 75 (PASI75) response; percent change from baseline in PASI and body surface area; change from baseline in Itch Severity Item (ISI). Adverse events (AEs) were monitored and clinical laboratory parameters measured.

Results: Overall, 435 patients were randomized and 430 patients received treatment. The proportion of patients with PGA-C clear or almost clear and $\geq 2$ grade improvement from baseline at Week 8 was $18.6 \%$ for $2 \%$ tofacitinib QD (80\% confidence interval [CI] for difference from vehicle: 3.8, 18.2 \%) and $22.5 \%$ for $2 \%$ tofacitinib BID (80\% Cl: 3.1, $18.5 \%)$; this was significantly higher vs vehicle for both dosage regimens. No significant difference vs vehicle was seen at Week 12. Significantly more patients achieved PGA-C clear or almost clear with $2 \%$ tofacitinib QD and BID and $1 \%$ tofacitinib QD (not BID) at Week 8, and with $2 \%$ tofacitinib BID at Week 12. Pruritus was significantly reduced vs vehicle with $2 \%$ and $1 \%$ tofacitinib BID (starting Day 2), and $2 \%$ tofacitinib QD (starting Day 3). Overall, $44.2 \%$ of patients experienced AEs, $8.1 \%$ experienced application site AEs, and $2.3 \%$ experienced serious AEs. The highest incidence of AEs (including application site AEs) was in the vehicle QD group.
\end{abstract}

Conclusions: In adults with mild to moderate plaque psoriasis, $2 \%$ tofacitinib ointment QD and BID showed greater efficacy than vehicle at Week 8 , but not Week 12 , with an acceptable safety and local tolerability profile.

Trial registration: NCT01831466 registered March 28, 2013.

Keywords: Psoriasis, Topical, Tofacitinib, CP-690,550, Physician's Global Assessment, Psoriasis Area and Severity Index, PASI, Dermatology Life Quality Index, Pruritus, Itch

\footnotetext{
* Correspondence: william.c.ports@pfizer.com

${ }^{8}$ Pfizer Worldwide Biopharmaceuticals, Global Innovative Pharma Business,

Groton, CT, USA

Full list of author information is available at the end of the article
} 


\section{Background}

The World Health Organization has described psoriasis as a 'chronic, non-communicable, painful, disfiguring and disabling disease for which there is no cure' [1]. The majority of people with plaque psoriasis (75-90\%) are considered to have relatively limited mild to moderate disease [2, 3]. Many treatments are available for mild to moderate psoriasis, including topical treatment with corticosteroids, often in combination with vitamin $\mathrm{D}$ analogues [4-6]. The use of mid to high potency corticosteroids can be limited by local and systemic adverse effects, particularly on the face and intertriginous areas $[5,7,8]$. Irritation or burning can also occur with vitamin D analogues [9-11]. Topical therapy is also used in combination with phototherapy or systemic therapy in patients with moderate to severe psoriasis [12].

A substantial proportion of patients with psoriasis are dissatisfied with their current treatment [13]. The limited efficacy of non-steroidal topical monotherapy or low potency corticosteroids and the safety issues associated with long-term use of mid to high potency topical corticosteroids suggest an unmet need exists for additional topical therapeutic options.

Tofacitinib (CP-690,550) is a small molecule Janus kinase (JAK) inhibitor; inhibition of JAK1 and JAK3 by tofacitinib blocks signaling of multiple cytokines implicated in immune response and inflammation. The oral formulation of tofacitinib is effective in patients with moderate to severe plaque psoriasis [14-16]. An ointment formulation of tofacitinib investigated for the topical treatment of psoriasis in a Phase $2 \mathrm{a}$ study showed the ointment ( $2 \%$ twice daily [BID]) was effective with acceptable tolerability for mild to moderate psoriasis [17].

The primary objective of this Phase $2 \mathrm{~b}$ study was to further characterize the efficacy and safety of tofacitinib ointment (2\% and $1 \%$ ) applied once daily (QD) or BID over 12 weeks in adult patients with mild or moderate chronic plaque psoriasis, compared with the corresponding vehicle.

\section{Methods}

\section{Study design and treatment}

This randomized, double-blind, parallel-group, vehiclecontrolled study (NCT01831466), conducted at 52 centers in the United States, Canada, Denmark, and Poland, was initiated in May 2013 and completed in September 2014. Patients were randomized $1: 1: 1$ to receive $1 \%$ (10 mg/g) tofacitinib ointment, $2 \%(20 \mathrm{mg} / \mathrm{g})$ tofacitinib ointment or corresponding vehicle. Randomization was stratified by baseline severity of psoriasis as defined by the Calculated Physician's Global Assessment (PGA-C). Investigators, study staff and sponsor remained blinded to treatment and randomization information until after the conclusion of the study. Investigator sites were assigned to either QD or BID regimen, but not both; neither investigators nor patients were blinded to regimen.

Tofacitinib ointment was provided in $60 \mathrm{~g}$ tubes at a strength of $2 \%$ (maximum feasible concentration) and $1 \%$; the matching vehicle contained the same inactive ingredients as tofacitinib ointment. Treatments were administered topically at a target application coverage of $3 \mathrm{mg} / \mathrm{cm}^{2}$ to a treatment area corresponding to 2 to $20 \%$ of the patient's body surface area (BSA). Patients were instructed to treat all treatment-eligible psoriatic areas identified at baseline for 12 weeks, regardless of clearing or improvement in psoriasis. On study visit days, showering or bathing, but not moisturizing, was permitted prior to attending, and study drug was applied in the clinic after study assessments were completed. After the final study treatment, the treatment areas were left untreated during the 4-week follow-up period.

Use of shampoo containing tar, salicylic acid or low or least potent corticosteroid products (eg hydrocortisone and hydrocortisone acetate $\leq 1 \%$ ) was permitted on hairbearing scalp only throughout the study. The proprietary ointment formulation contained standard excipients for a topical formulation.

\section{Patients \\ Key inclusion criteria}

Subjects were aged $\geq 18$ years with chronic plaque psoriasis for $\geq 6$ months, were required to have a PGA-C score of mild (2) or moderate (3), and have plaque psoriasis covering $2-20 \%$ of their BSA on the trunk and/or limbs, with $\geq 1 \%$ BSA involvement on the trunk and/or limbs (excluding palms, soles, elbows, knees and below the knees).

\section{Key exclusion criteria}

Exclusion criteria included non-plaque forms of psoriasis; drug-induced psoriasis; evidence of skin conditions that would interfere with the evaluation of psoriasis; history of infection requiring hospitalization or treatment with oral or topical antimicrobial therapy within 2 weeks prior to baseline; hepatitis $\mathrm{B} / \mathrm{C}$ or HIV infection; history of lymphoproliferative disorder or malignancy, except adequately treated or excised basal/squamous cell carcinoma, or cervical carcinoma in situ; evidence of tuberculosis infection; treatment with ustekinumab within the previous 4 months or other biologic agents (excluding etanercept) within the previous 2 months; phototherapy or treatment with etanercept or conventional systemic treatments that could affect psoriasis, such as oral or injectable corticosteroids, retinoids, methotrexate, and cyclosporine, within 4 weeks prior to the first study dose. 
Topical treatments that could affect psoriasis (eg corticosteroids, tars, keratolytics, anthralin, vitamin D analogues, and retinoids) were discontinued for $\geq 2$ weeks prior to the first study dose.

\section{Assessments}

Clinical signs of plaque psoriasis (erythema, induration and scaling) were scored separately according to a 5-point severity scale: clear (0), almost clear (1), mild (2), moderate (3), and severe (4). These PGA subscores were then summed, averaged, and rounded to the nearest whole number to determine the PGA-C score and category [18]. Evaluation of the PGA-C excluded the scalp (even if the hairless scalp was being treated with study drug), palms, soles, and nails.

The primary endpoint was the proportion of subjects achieving a PGA-C response of clear (0) or almost clear (1) with $\geq 2$ grade improvement from baseline at Week 8 and Week 12, independently. Secondary endpoints included Week 8 and Week 12 assessments of the proportion of patients achieving a PGA-C response of clear (0) or almost clear (1); the proportion of patients achieving a $\geq 75 \%$ improvement from baseline in Psoriasis Area and Severity Index (PASI75); the percent change from baseline in PASI; and the percent change from baseline in affected BSA.

Evaluation of patient-reported outcomes included change from baseline in itch severity and in the Dermatology Life Quality Index (DLQI). The severity of itch was assessed via the Itch Severity Item (ISI), a single item instrument in which the patient records itching over the previous $24 \mathrm{~h}$ on a numerical rating scale of 0 (no itching) to 10 (worst possible itching) [19]. ISI was recorded in the clinic during Visit 1 (baseline/Day 1) and at Visits 3-7 (Weeks 2, 4, 8, 12 , and 16), as well as once per day between Visit 1 and the day before Visit 3 by the patient in a diary prior to application of study treatment. Patients in the BID treatment group recorded the ISI before applying either the morning or evening treatment, but at the same time throughout this period.

Safety endpoints included the incidence of treatmentemergent adverse events (AEs), serious AEs (SAEs), and application site AEs, plus the proportion of patients who discontinued due to application site AEs. Physical examination, monitoring of vital signs, and clinical laboratory assessments (including hematology, fasting serum chemistry, fasting lipid panels, and urinalysis) were performed.

Pharmacokinetic (PK) endpoints included tofacitinib PK concentrations for pre-dose and post-dose samples. Pre-dose blood samples were collected at baseline and at Weeks 2, 4, 8, and $12(0 \mathrm{~h})$. At selected sites, three PK samples were also collected at Week 4 post-dose between $30 \mathrm{~min}$ and $1 \mathrm{~h}$, between 2 and $3 \mathrm{~h}$, and between 4 and $10 \mathrm{~h}$.

\section{Statistical analysis}

This was an estimation study. A sample size of 70 subjects per treatment group was selected, such that the $80 \%$ confidence interval (CI) width of the difference between tofacitinib and vehicle was approximately $19 \%$, assuming a $21 \%$ vehicle response and a $36 \%$ response in tofacitinib. Additionally, this sample size would yield approximately $76 \%$ power to establish the superiority of each strength and regimen of tofacitinib to its respective vehicle for the primary endpoint at the 0.10 (one-sided) significance level. No adjustment for multiple comparisons was made.

Patients with mild or moderate psoriasis at baseline (as defined by PGA-C) who were randomized and received at least one dose of study medication (tofacitinib or vehicle) were included in the analyses. Data at Week 8 and Week 12 were evaluated separately.

For the primary endpoint, standard error (SE) and two-sided $80 \%$ CI were calculated using the normal approximation to the binomial proportions. A stratified analysis was conducted by summarizing the difference in proportions adjusted for the baseline PGA-C disease severity using the Cochran-MantelHaenszel approach [20, 21]. Patients with missing values were considered non-responders.

PASI75 and PGA-C response of clear or almost clear were analyzed using a marginal logistic regression model fit by pseudo-likelihood (generalized linear mixed model for repeated measures). Response proportions were estimated from the model and odds ratios for treatment contrasts along with $80 \% \mathrm{CI}$ were determined. Continuous variables (eg percent change from baseline in PASI and BSA, and change from baseline in ISI) were analyzed using a linear mixed model for repeated measures. Least squares mean (LSM), difference in LSM, SE, and two-sided $80 \% \mathrm{CI}$ were calculated. All analyses used observed data without imputation. Separate models were fit for the QD and BID data.

For comparisons in response proportions between the active treatment and corresponding vehicle, statistical significance was declared if the lower limit of the twosided $80 \% \mathrm{CI}$ for the response difference was $>0$ for the primary efficacy endpoint, and if the lower limit of the two-sided $80 \% \mathrm{CI}$ for the odds ratio was $>1$ for the secondary PGA-C and PASI75 endpoints. For comparisons in LSMs between active treatment and corresponding vehicle, statistical significance was declared if the upper limit of the two-sided $80 \%$ CI was $<0$ for percent change from baseline in PASI and BSA and change from baseline in ISI. No adjustment for multiple comparisons was made. 
All statistical analyses were performed using SAS Software [22].

\section{Results}

\section{Patients}

Overall, 435 patients were randomized (Fig. 1). In the QD treatment groups, 218 patients received either $2 \%$ tofacitinib, $1 \%$ tofacitinib, or vehicle $(n=70,74,74$, respectively). In the BID treatment groups, 212 patients received either $2 \%$ tofacitinib, $1 \%$ tofacitinib, or vehicle ( $n=71,70,71$, respectively). Baseline demographics were generally similar across the treatment groups, with the exception of geographical distribution between the dosing regimens (Table 1$)$.

\section{Efficacy}

Only those treatment groups and time points that were statistically significant are described within the text.

\section{Primary endpoints}

At Week 8 only, significantly more patients receiving $2 \%$ tofacitinib QD and $2 \%$ tofacitinib BID achieved a PGA-C response of clear or almost clear and $\geq 2$ grade improvement from baseline compared with the corresponding vehicle. Response rate was $18.6 \%$ and $8.1 \%$ for $2 \%$ tofacitinib QD and vehicle QD, respectively, and $22.5 \%$ and $11.3 \%$ for $2 \%$ tofacitinib BID and vehicle BID, respectively. The difference $(80 \% \mathrm{CI})$ between response to active treatment and vehicle was $10.8 \%(3.1,18.5)$ and $11.0 \%(3.8,18.2)$ for $2 \%$ tofacitinib BID and QD administration, respectively (Fig. 2a-b). At Week 12 , no statistically significant differences versus vehicle were seen for $2 \%$ or $1 \%$ tofacitinib by either dosing regimen (Fig. 2a-b).

\section{Secondary endpoints}

The proportion of patients achieving a PGA-C response of clear or almost clear was significantly greater for the $2 \%$ tofacitinib QD (35.9\%), $2 \%$ tofacitinib BID (41.8\%) and $1 \%$ tofacitinib QD (23.4\%) treatment groups compared with vehicle (QD $13.8 \%$, BID $25.2 \%$ ) at Week 8, and for the $2 \%$ tofacitinib BID (39.7\%) treatment group compared with vehicle (27.3\%) at Week 12 (Table 2).

At Week 8 and Week 12, significantly more patients receiving $2 \%$ tofacitinib QD $(17.9 \%$ and $23.0 \%$, respectively) achieved a PASI75 response vs vehicle ( $8.3 \%$ and $8.8 \%$, respectively) (Table 2 ). The percent change from baseline in PASI was also significantly greater for the $2 \%$ tofacitinib QD treatment group compared with vehicle at Week 8 and Week 12 (Table 2); the differences $(80 \% \mathrm{CI})$ vs corresponding vehicle were $-9.2 \%(-17.1$, $-1.4)$ and $-12.3 \%(-21.8,-2.8)$ at Weeks 8 and 12, respectively. The percent change from baseline in BSA was also significantly greater for the $2 \%$ tofacitinib QD treatment group compared with vehicle at Week 12 (Table 2$)$; the difference $(80 \% \mathrm{CI})$ vs corresponding vehicle was $-20.0 \%(-31.4,-8.7)$.

\section{Patient-reported outcomes}

$2 \%$ and $1 \%$ tofacitinib BID significantly reduced pruritus compared with vehicle BID as early as Day 2 (the day following the initial dose); these improvements were sustained through Day 14 (Fig. 3a). Numerically greater improvements in ISI were also seen in the $2 \%$ and $1 \%$ tofacitinib QD treatment groups compared with vehicle QD; these improvements were statistically significant for $2 \%$ tofacitinib QD on Days 3-14 (Fig. 3b). Significant improvements in pruritus were maintained for $2 \%$ BID, $1 \%$ BID, and $2 \%$ QD from Week 2 through Week 12 (except Week 8 and 12 for $2 \%$ QD).

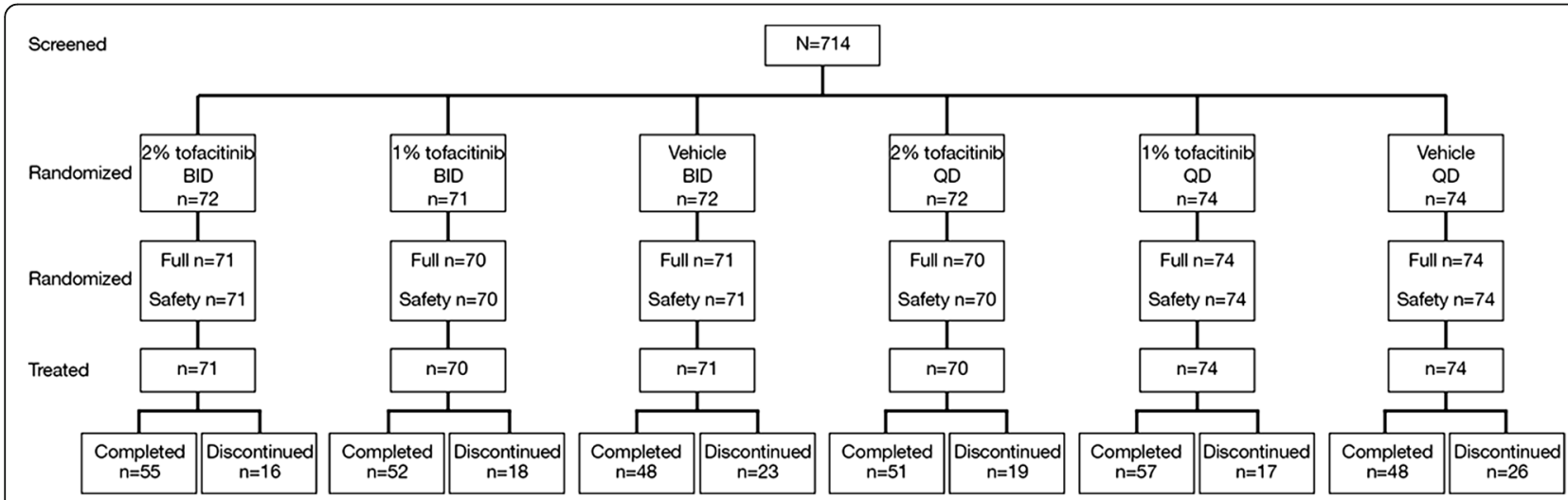

Fig. 1 Patient disposition. Note that the 714 subjects includes subjects with mild, moderate, and severe psoriasis; subjects randomized are those that met the mild to moderate psoriasis eligibility criteria. Following database release, it was discovered that five subjects were randomized, but did not have any record of study drug dosing and were assessed in the clinical database as non-treated subjects; as a consequence, these subjects were excluded from all analyses. BID twice daily, QD once daily 
Table 1 Baseline patient demographics and disease characteristics

\begin{tabular}{|c|c|c|c|c|c|c|}
\hline & $2 \%$ tofacitinib BID & $1 \%$ tofacitinib BID & Vehicle BID & $2 \%$ tofacitinib QD & $1 \%$ tofacitinib QD & Vehicle QD \\
\hline & $N=71$ & $N=70$ & $N=71$ & $N=70$ & $N=74$ & $N=74$ \\
\hline \multicolumn{7}{|l|}{ Age (years) } \\
\hline Mean (SD) & $47.6(15.6)$ & $50.4(14.5)$ & $48.8(15.0)$ & $50.7(13.2)$ & $47.8(14.0)$ & $48.9(13.9)$ \\
\hline Range & $18.0-74.0$ & $18.0-77.0$ & $21.0-84.0$ & $21.0-77.0$ & $20.0-85.0$ & $20.0-74.0$ \\
\hline Male (\%) & 60.6 & 67.1 & 57.7 & 52.9 & 67.6 & 56.8 \\
\hline \multicolumn{7}{|l|}{$\mathrm{BMI}\left(\mathrm{kg} / \mathrm{m}^{2}\right)$} \\
\hline Mean (SD) & $31.8(7.9)$ & $29.6(5.6)$ & $30.2(8.3)$ & $28.9(7.8)$ & $31.0(7.2)$ & $31.1(6.5)$ \\
\hline Range & $17.2-58.4$ & $19.2-51.4$ & $17.0-79.8$ & $16.4-68.6$ & $17.6-50.4$ & $20.6-47.4$ \\
\hline \multicolumn{7}{|l|}{ Race (\%) } \\
\hline White & 93.0 & 85.7 & 94.4 & 90.0 & 91.9 & 97.3 \\
\hline Black & 1.4 & 4.3 & 1.4 & 1.4 & 2.7 & 2.7 \\
\hline Asian & 4.2 & 7.1 & 2.8 & 5.7 & 2.7 & 0.0 \\
\hline Other & 1.4 & 2.9 & 1.4 & 2.9 & 2.7 & 0.0 \\
\hline \multicolumn{7}{|c|}{ Geographical region (\%) } \\
\hline Canada & 15.5 & 30.0 & 26.8 & 27.1 & 23.0 & 33.8 \\
\hline Denmark & 1.4 & 1.4 & 0.0 & 1.4 & 1.4 & 1.4 \\
\hline Poland & 14.1 & 12.9 & 15.5 & 32.9 & 39.2 & 28.4 \\
\hline United States & 69.0 & 55.7 & 57.7 & 38.6 & 36.5 & 36.5 \\
\hline \multicolumn{7}{|l|}{ PGA-C (\%) } \\
\hline Mild & 28.2 & 30.0 & 29.6 & 32.9 & 27.0 & 27.0 \\
\hline Moderate & 71.8 & 70.0 & 70.4 & 67.1 & 73.0 & 73.0 \\
\hline \multicolumn{7}{|l|}{ PASI score } \\
\hline Mean (SD) & $9.5(5.1)$ & $8.5(3.3)$ & 8.5 (3.6) & $9.9(4.1)$ & $10.1(4.4)$ & $9.6(3.8)$ \\
\hline Range & $2.4-29.0$ & $3.0-18.0$ & $2.4-18.0$ & $2.0-19.8$ & $2.8-19.8$ & $3.2-17.1$ \\
\hline \multicolumn{7}{|l|}{ BSA (\%) } \\
\hline Mean (SD) & $7.6(4.6)$ & $6.4(3.8)$ & $6.5(4.1)$ & $7.8(4.3)$ & $8.4(4.9)$ & $8.0(4.5)$ \\
\hline Range & $2.0-19.0$ & $1.5-17.0$ & $2.0-20.0$ & $2.0-19.0$ & $2.4-20.0$ & $2.0-19.0$ \\
\hline \multicolumn{7}{|l|}{ ISI score ${ }^{a}$} \\
\hline Mean (SD) & $5.8(2.6)$ & $5.3(2.4)$ & $5.4(2.6)$ & $6.0(2.7)$ & $5.7(2.9)$ & $5.4(3.0)$ \\
\hline Range & $0.0-10.0$ & $1.0-10.0$ & $0.0-10.0$ & $0.0-10.0$ & $0.0-10.0$ & $0.0-10.0$ \\
\hline \multicolumn{7}{|l|}{ DLQI } \\
\hline Mean (SD) & $10.6(5.9)$ & $8.6(5.5)$ & $9.3(6.0)$ & $12.2(7.4)$ & $10.9(7.0)$ & $10.2(6.5)$ \\
\hline Range & $0.0-25.0$ & $1.0-25.0$ & $1.0-24.0$ & $1.0-29.0$ & $1.0-29.0$ & $0.0-26.0$ \\
\hline
\end{tabular}

${ }^{a}$ Two patients were missing baseline ISI scores (1 in $2 \%$ tofacitinib QD; 1 in $1 \%$ tofacitinib BID)

$B I D$ twice daily, BMI body mass index, BSA body surface area, DLQI Dermatology Life Quality Index, ISI Itch Severity Item, PASI Psoriasis Area and Severity Index, $P G A-C$ Calculated Physician's Global Assessment, QD once daily, SD standard deviation

At Week 8, 2 \% tofacitinib BID and QD significantly improved DLQI more than their respective vehicles (Additional file 1: Figure S1). At Week 12, $2 \%$ tofacitinib QD and $1 \%$ tofacitinib QD significantly improved DLQI more than the vehicle (Additional file 1: Figure S1).

\section{Safety}

All adverse events

Overall, $44.2 \%$ of patients experienced treatmentemergent AEs, most of which were mild or moderate in severity (Table 3). The highest incidence of treatmentemergent AEs was in the vehicle QD group, with $54.1 \%$ of patients in this group reporting one or more treatment-emergent AE. The most frequently reported AEs by Medical Dictionary for Regulatory Activities (MedDRA; version 17.1) preferred term were nasopharyngitis (6.7\%), upper respiratory tract infection (4.9\%), and psoriasis $(4.9 \%)$.

A total of 11 SAEs were experienced by $10(2.3 \%)$ patients. No SAEs were reported in the $2 \%$ tofacitinib 

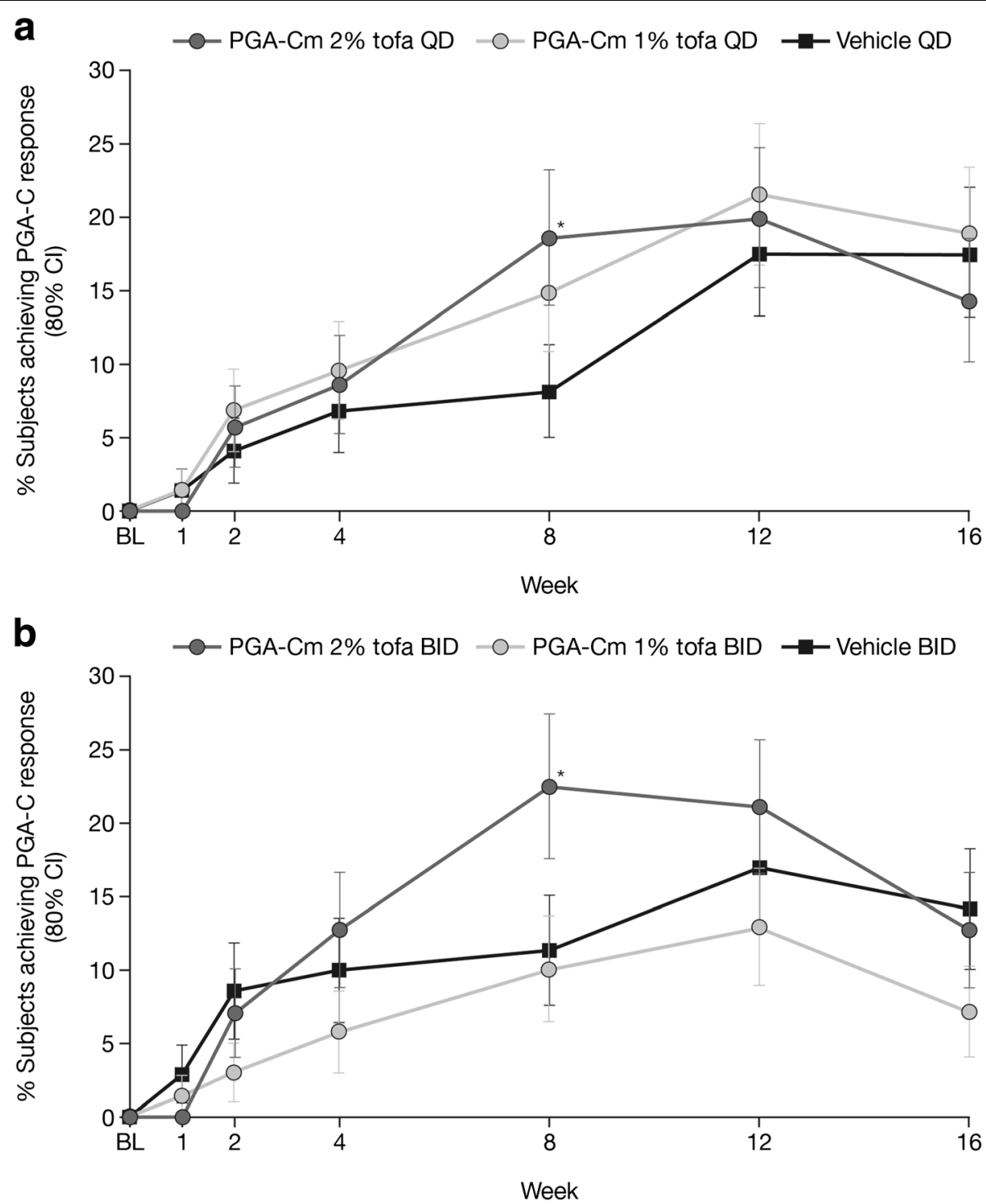

Fig. 2 PGA-C response of clear (0)/almost clear (1) and $\geq 2$ grade improvement at Week 16. *Lower limit $80 \% \mathrm{Cl}$ of difference tofacitinib versus vehicle $>0$. Proportion (SE) of patients achieving a PGA-C response of clear (0) or almost clear (1) and $\geq 2$ grade improvement from baseline through to Week 16 for patients applying $2 \%$ tofacitinib, $1 \%$ tofacitinib, or vehicle, once daily (a) or twice daily (b). Patients who were discontinued or with missing values were considered non-responders. BID twice daily, BL baseline, $C I$ confidence interval, $P G A-C$ Calculated Physician's Global Assessment, PGA-Cm Calculated Physician's Global Assessment of patients with mild to moderate plaque psoriasis at baseline, QD once daily, SE standard error, tofa tofacitinib

QD or BID treatment groups; SAEs were reported in five patients in the $1 \%$ tofacitinib BID group, in two patients in each of the $1 \%$ tofacitinib QD and vehicle BID groups, and in one patient in the vehicle QD group (Additional file 1: Table S1). No SAEs were assessed by the investigator as treatment-related, with the exception of one SAE of psoriatic arthropathy in the vehicle BID treatment group.

Overall, 21 (4.9\%) patients discontinued from the study due to AEs, most commonly psoriasis, which was reported by six $(1.4 \%)$ patients. Seven patients discontinued due to AEs in the vehicle QD group, six with $2 \%$ tofacitinib QD, four with vehicle BID, three with $1 \%$ tofacitinib QD, and one with $1 \%$ tofacitinib BID (patient was discontinued from the study due to a fatal myocardial infarction as described below).

One death (due to myocardial infarction) occurred in a 53-year-old white male receiving $1 \%$ tofacitinib BID. His final application of tofacitinib ointment was on Study Day 74 and he died on Study Day 86. Relevant medical history included prior myocardial infarction and stent placement, dyslipidemia, hypertension, and 18-year history of tobacco use. The event was considered unrelated to study treatment by the investigator. 
Table 2 Secondary efficacy endpoints

\begin{tabular}{|c|c|c|c|c|c|c|c|}
\hline Endpoint & Week & $2 \%$ tofacitinib BID & $1 \%$ tofacitinib BID & Vehicle BID & $2 \%$ tofacitinib QD & $1 \%$ tofacitinib QD & Vehicle QD \\
\hline \multirow{2}{*}{$\begin{array}{l}\text { PGA-C, clear }(0) \\
\text { or almost clear }(1) \\
\text { Responders, } \%(n / N) \\
\text { Odds ratios }(80 \% \mathrm{Cl})\end{array}$} & 8 & $\begin{array}{l}41.8(26 / 61) \\
2.13^{\mathrm{b}}(1.29,3.54)\end{array}$ & $\begin{array}{l}20.9(14 / 64) \\
0.79(0.45,1.36)\end{array}$ & $25.2(16 / 55)$ & $\begin{array}{l}35.9(23 / 60) \\
3.52^{\mathrm{b}}(1.97,6.28)\end{array}$ & $\begin{array}{l}23.4(16 / 67) \\
1.92^{\mathrm{b}}(1.06,3.48)\end{array}$ & $13.8(9 / 58)$ \\
\hline & 12 & $\begin{array}{l}39.7(24 / 58) \\
1.75^{\mathrm{b}}(1.06,2.90)\end{array}$ & $\begin{array}{l}28.4(18 / 57) \\
1.06(0.62,1.78)\end{array}$ & $27.3(17 / 55)$ & $\begin{array}{l}36.1(19 / 53) \\
1.36(0.81,2.28)\end{array}$ & $\begin{array}{l}32.9(22 / 62) \\
1.18(0.71,1.97)\end{array}$ & $29.3(17 /$ \\
\hline \multirow{2}{*}{$\begin{array}{l}\text { PASI75 } \\
\text { Responders, \% (n/N) } \\
\text { Odds ratios (80\% Cl) }\end{array}$} & 8 & $\begin{array}{l}15.2(10 / 61) \\
2.06(0.96,4.45)\end{array}$ & $\begin{array}{l}9.1(6 / 64) \\
1.15(0.50,2.66)\end{array}$ & $8.0(5 / 55)$ & $\begin{array}{l}17.9(11 / 60) \\
2.40^{\mathrm{b}}(1.14,5.05)\end{array}$ & $\begin{array}{l}7.2(5 / 67) \\
0.85(0.36,2.01)\end{array}$ & $8.3(5 / 58)$ \\
\hline & 12 & $\begin{array}{l}20.3(12 / 58) \\
1.42(0.76,2.64)\end{array}$ & $\begin{array}{l}14.4(9 / 57) \\
0.94(0.48,1.83)\end{array}$ & $15.2(9 / 55)$ & $\begin{array}{l}23.0(11 / 53) \\
3.11^{\mathrm{b}}(1.47,6.55)\end{array}$ & $\begin{array}{l}12.1(8 / 62) \\
1.44(0.65,3.19)\end{array}$ & $8.8(5 / 52)$ \\
\hline \multirow{2}{*}{ 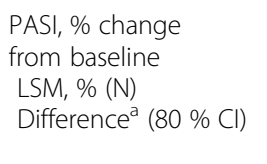 } & 8 & $\begin{array}{l}-31.8(61) \\
-8.3(-17.3,0.6)\end{array}$ & $\begin{array}{l}-26.7(64) \\
-3.2(-12.1,5.7)\end{array}$ & $-23.5(55)$ & $\begin{array}{l}-28.3(60) \\
-9.2^{b}(-17.1,-1.4)\end{array}$ & $\begin{array}{l}-25.5(67) \\
-6.4(-14.1,1.3)\end{array}$ & $-19.1(58)$ \\
\hline & 12 & $\begin{array}{l}-33.9(58) \\
-6.5(-17.1,4.1)\end{array}$ & $\begin{array}{l}-32.6(57) \\
-5.2(-15.7,5.3)\end{array}$ & $-27.4(55)$ & $\begin{array}{l}-33.4(53) \\
-12.3^{b}(-21.8,-2.8)\end{array}$ & $\begin{array}{l}-27.0(62) \\
-5.9(-15.2,3.3)\end{array}$ & $-21.1(52)$ \\
\hline \multirow{2}{*}{$\begin{array}{l}\text { BSA, \% change } \\
\text { from baseline } \\
\text { LSM, \% (N) } \\
\text { Difference }^{a}(80 \% \text { Cl) }\end{array}$} & 8 & $\begin{array}{l}-22.1(61) \\
-4.2(-13.1,4.7)\end{array}$ & $\begin{array}{l}-20.9(64) \\
-3.0(-11.8,5.8)\end{array}$ & $-17.9(55)$ & $\begin{array}{l}-12.5(60) \\
-8.0(-16.7,0.7)\end{array}$ & $\begin{array}{l}-10.1(67) \\
-5.7(-14.2,2.8)\end{array}$ & $-4.5(58)$ \\
\hline & 12 & $\begin{array}{l}-31.2(58) \\
-4.5(-13.8,4.9)\end{array}$ & $\begin{array}{l}-26.0(57) \\
0.8(-8.5,10.0)\end{array}$ & $-26.7(55)$ & $\begin{array}{l}-22.8(53) \\
-20.0^{\mathrm{b}}(-31.4,-8.7)\end{array}$ & $\begin{array}{l}-12.3(62) \\
-9.5(-20.6,1.6)\end{array}$ & $-2.8(52)$ \\
\hline
\end{tabular}

${ }^{a}$ Difference active - vehicle; ${ }^{b}$ meets specification for statistical significance

PASI excluded the scalp, palms, and soles from the assessment/scoring, even if these areas were being treated with study drug. BSA excluded the head, neck, palms, and soles, even if these areas were being treated with study drug

PGA-C and PASI75 responses were analyzed using a Generalized Mixed Model for Repeated Measures without imputation for missing values; percent changes from baseline in PASI and BSA were analyzed using a Mixed Model for Repeated Measures without imputation for missing values; QD and BID data were analyzed separately

BID twice daily, BSA body surface area, Cl confidence interval, LSM least squares mean, PASI Psoriasis Area and Severity Index, PGA-C Calculated Physician's Global Assessment, $Q D$ once daily

\section{Application site adverse events}

Application site AEs were reported in 35 of the 190 (18.4 \%) patients who experienced treatmentemergent AEs (8.1\% of total study population); no application site AEs were serious (Table 3). The highest incidence was in the vehicle QD group (Table 3). The most frequently reported application site AEs by MedDRA preferred term were psoriasis (reported by 18 [4.2\%] patients), pruritus (9 [2.1\%]), and application site pain (3 [0.7\%]).

A total of $12(2.8 \%)$ patients discontinued the study due to application site AEs; seven were from the vehicle QD group, three from the $2 \%$ tofacitinib QD group, and one from each of the vehicle BID and $1 \%$ tofacitinib QD groups. The most common application site AE leading to discontinuation was psoriasis, which was reported by six $(1.4 \%)$ patients.

\section{Laboratory assessments}

Thirteen patients met the criteria for laboratory safety monitoring (Table 4); no patients met the laboratory monitoring criteria for discontinuation.

\section{Pharmacokinetics}

Tofacitinib concentrations were above the lower limit of quantification of $0.01 \mathrm{ng} / \mathrm{mL}$ in most plasma samples, with the largest percentage of samples in the concentration range of 0.1 to $<1.0 \mathrm{ng} / \mathrm{mL}$ (Additional file 1: Table $\mathrm{S} 2)$. There was a general trend toward higher concentrations with higher dose strength ( $2 \%$ vs $1 \%$ ) but no clear difference between the dosing regimens (BID vs QD). Across tofacitinib treatment groups, $83.3 \%-97.4 \%$ of plasma tofacitinib concentrations were $<1.0 \mathrm{ng} / \mathrm{mL}$. The maximum observed plasma concentration of $9.7 \mathrm{ng} / \mathrm{mL}$ occurred at Week 12 in the $2 \%$ tofacitinib QD group. Based on the post-dose PK obtained in a limited number of patients, the PK had a flat profile with limited fluctuation in concentrations between doses, as would be expected after topical application. Total exposure based on area under the plasma concentration time profile from time zero to the time tau $\left(\mathrm{AUC}_{\mathrm{tau}}\right)$ in patients with post-dose PK was higher with the higher dose strength, while the relationship between exposure and dose regimen was not clear (Additional file 1: Table S3).

\section{Discussion}

Partial inhibition of JAK signaling by tofacitinib results in a multi-tiered intervention in the cycle of psoriasis pathogenesis, with direct impact on dysregulated keratinocytes, reduction in inflammatory infiltrate and, ultimately, normalization of the interleukin (IL)-23/Th17 axis [23]. Oral tofacitinib is effective in patients with moderate to severe plaque psoriasis [14-16], and evidence of efficacy has been seen in the patient with mild to moderate plaque psoriasis with a $2 \%$ topical formulation of tofacitinib applied BID [17].

The current study assessed the efficacy and safety of two dose strengths (2\% and $1 \%$ ) of tofacitinib ointment applied either QD or BID in adult patients with mild to moderate plaque psoriasis. Greater efficacy response was 

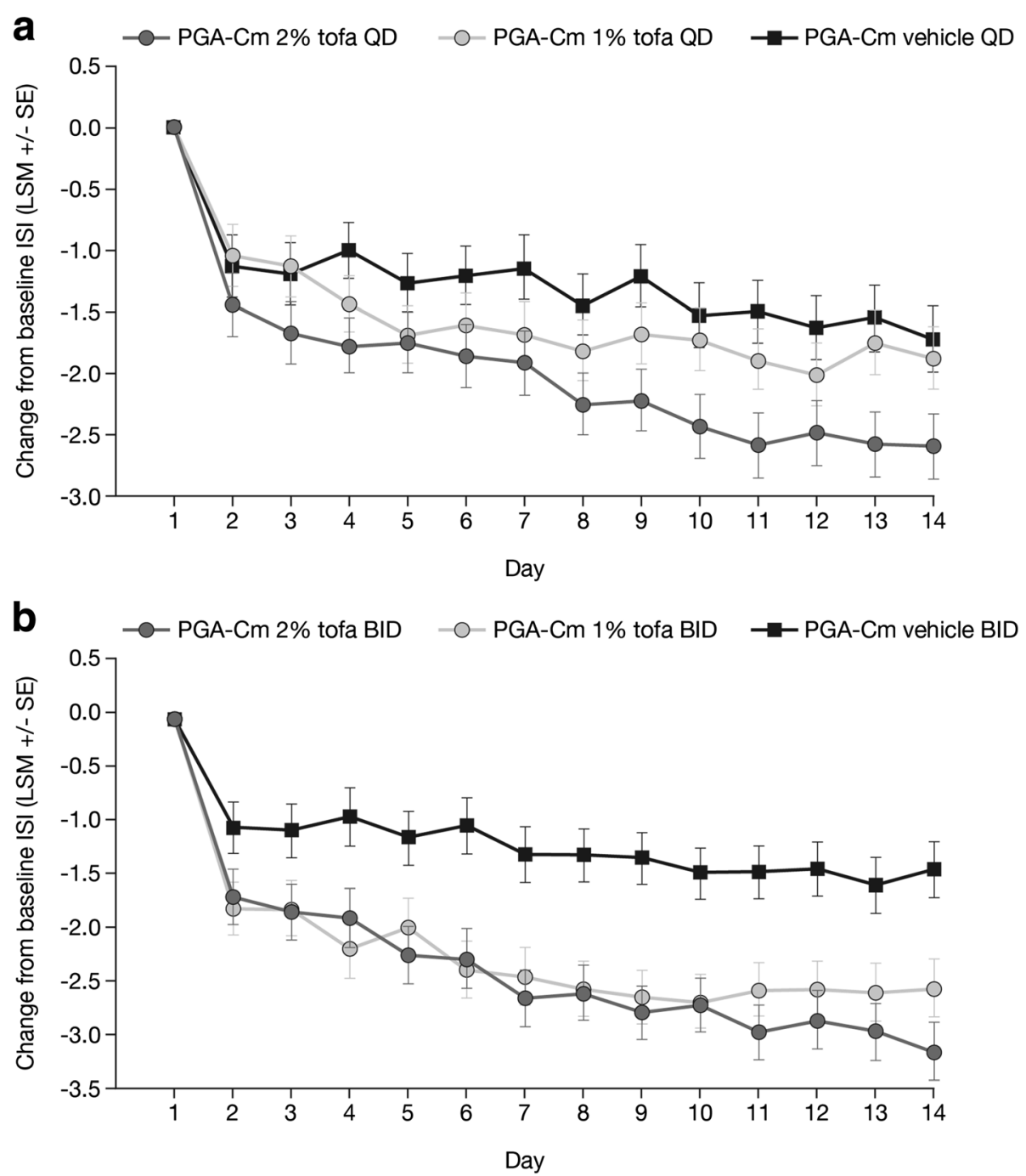

Fig. 3 Change from baseline in Itch Severity Item score through Week 2. Least squares mean (SE) change from baseline in Itch Severity Item score through Week 2 for patients applying $2 \%$ tofacitinib, $1 \%$ tofacitinib, or vehicle, once daily (a) or twice daily (b). Changes from baseline in ISI were analyzed using a Mixed Model for Repeated Measures without imputation for missing values; QD and BID data were analyzed separately. BID twice daily, ISI Itch Severity Item, LSM least squares mean, PGA-Cm Calculated Physician's Global Assessment of patients with mild to moderate plaque psoriasis at baseline, QD once daily, SE standard error, tofa tofacitinib

Table 3 Summary of adverse events, patients discontinued due to adverse events and deaths

\begin{tabular}{|c|c|c|c|c|c|c|}
\hline & $2 \%$ tofacitinib BID & $1 \%$ tofacitinib BID & $\begin{array}{l}\text { Vehicle } \\
\text { BID }\end{array}$ & $2 \%$ tofacitinib QD & $1 \%$ tofacitinib QD & $\begin{array}{l}\text { Vehicle } \\
\text { QD }\end{array}$ \\
\hline & $N=71$ & $N=70$ & $N=71$ & $N=70$ & $N=74$ & $N=74$ \\
\hline Number of AEs & 47 & 51 & 54 & 66 & 65 & 62 \\
\hline Patients with treatment-emergent AEs, n (\%) & $30(42.3)$ & $30(42.9)$ & $28(39.4)$ & $34(48.6)$ & $28(37.8)$ & $40(54.1)$ \\
\hline Patients with application site AEs, n (\%) & $4(5.6)$ & $0(0.0)$ & $4(5.6)$ & $8(11.4)$ & $7(9.5)$ & $12(16.2)$ \\
\hline Patients with SAEs, n (\%) & $0(0.0)$ & $5(7.1)$ & $2(2.8)$ & $0(0.0)$ & $2(2.7)$ & $1(1.4)$ \\
\hline Patients discontinued due to AEs, n (\%) & $0(0.0)$ & $1(1.4)^{\mathrm{a}}$ & $4(5.6)$ & $6(8.6)$ & $3(4.1)$ & $7(9.5)$ \\
\hline Deaths, n (\%) & $0(0.0)$ & $1(1.4)^{\mathrm{a}}$ & $0(0.0)$ & $0(0.0)$ & $0(0.0)$ & $0(0.0)$ \\
\hline
\end{tabular}

${ }^{a}$ Patient had an AE of myocardial infarction and subsequently died; the patient is counted as a discontinuation due to AE and as a death Categories of adverse events experienced include treatment-emergent, application site and serious adverse events $A E$ adverse event, $B I D$ twice daily, $Q D$ once daily, SAE serious adverse event 
Table 4 Patients with laboratory values meeting pre-specified protocol criteria* for safety monitoring

\begin{tabular}{|c|c|c|c|c|c|c|}
\hline Criterion, \% (n/N) & $2 \%$ tofacitinib BID & $1 \%$ tofacitinib BID & $\begin{array}{l}\text { Vehicle } \\
\text { BID } \\
\end{array}$ & $2 \%$ tofacitinib QD & $1 \%$ tofacitinib QD & $\begin{array}{l}\text { Vehicle } \\
\text { QD }\end{array}$ \\
\hline Any criterion & $2.9(2 / 70)$ & $2.9(2 / 70)$ & $1.4(1 / 69)$ & $5.8(4 / 69)$ & $4.1(3 / 73)$ & $1.4(1 / 73)$ \\
\hline Hemoglobin $^{a}$ & $1.5(1 / 68)$ & $0.0(0 / 70)$ & $0.0(0 / 69)$ & $0.0(0 / 67)$ & $4.1(3 / 73)$ & $1.4(1 / 72)$ \\
\hline Neutrophil count ${ }^{\mathrm{b}}$ & $0.0(0 / 68)$ & $0.0(0 / 69)$ & $0.0(0 / 69)$ & $1.5(1 / 67)$ & $0.0(0 / 73)$ & $0.0(0 / 72)$ \\
\hline Lymphocyte count $^{c}$ & $0.0(0 / 68)$ & $0.0(0 / 70)$ & $0.0(0 / 69)$ & $0.0(0 / 67)$ & $0.0(0 / 73)$ & $0.0(0 / 71)$ \\
\hline Platelet count $^{\mathrm{d}}$ & $0.0(0 / 68)$ & $0.0(0 / 69)$ & $0.0(0 / 69)$ & $0.0(0 / 67)$ & $0.0(0 / 73)$ & $0.0(0 / 72)$ \\
\hline Serum creatinine $e^{e}$ & $1.4(1 / 69)$ & $0.0(0 / 70)$ & $0.0(0 / 69)$ & $1.5(1 / 67)$ & $0.0(0 / 73)$ & $0.0(0 / 72)$ \\
\hline AST/ALT ${ }^{f}$ & $0.0(0 / 69)$ & $2.9(2 / 70)$ & $1.4(1 / 69)$ & $1.5(1 / 67)$ & $0.0(0 / 73)$ & $0.0(0 / 72)$ \\
\hline $\mathrm{CPK}^{\mathrm{g}}$ & $0.0(0 / 69)$ & $0.0(0 / 70)$ & $1.4(1 / 69)$ & $3.0(2 / 67)$ & $0.0(0 / 73)$ & $0.0(0 / 72)$ \\
\hline
\end{tabular}

${ }^{*}$ a Any hemoglobin value $>2 \mathrm{~g} / \mathrm{dL}(>20 \mathrm{~g} / \mathrm{L})$ below baseline; ${ }^{\mathrm{b}}$ Absolute neutrophil count $<1.2 \times 10^{9} / \mathrm{L}\left(<1200 / \mathrm{mm}^{3}\right) ;{ }^{\mathrm{C}}$ Absolute lymphocyte count $<0.5 \times 10^{9} / \mathrm{L}$ $\left(<500\right.$ lymphocytes $\left./ \mathrm{mm}^{3}\right)$; d Platelet count $<100 \times 10^{9} / \mathrm{L}\left(<100,000 / \mathrm{mm}^{3}\right)$; ${ }^{\text {e}}$ Serum creatinine increase $>50 \%$ over the average of screening and baseline values OR absolute increase in serum creatinine $>0.5 \mathrm{mg} / \mathrm{dL}\left(>44.2 \mu \mathrm{mol} / \mathrm{L}\right.$ ) over the average of screening and baseline values; ${ }^{\mathrm{f}}$ Any AST and/or ALT elevation $\geq 3$ times the ULN, regardless of the total bilirubin; ${ }^{9}$ Any CPK $>5 x U L N$

$A L T$ alanine aminotransferase, AST aspartate aminotransferase, BID twice daily, CPK creatine phosphokinase, QD once daily, ULN upper limit of normal

generally observed with $2 \%$ tofacitinib than $1 \%$ tofacitinib, and overall no clear distinction in efficacy was seen between BID and QD dosing.

Greater efficacy of tofacitinib compared with vehicle was seen for more primary and secondary efficacy endpoints at Week 8 than Week 12. While it appeared that the PGA-C response of clear or almost clear and $\geq 2$ grade improvement from baseline plateaued after Week 8, the vehicle treatment group PGA-C responses continued to improve after Week 8, thereby decreasing the difference between tofacitinib and vehicle at Week 12. Explanation for the increase in vehicle responses between Week 8 and Week 12 was not evident after thorough review of the study data for potential contributing factors, although it is possible this could be related to the small sample size of the study.

Patients were required to achieve a $\geq 2$ grade improvement from baseline in PGA-C in addition to having a PGA-C score of clear or almost clear to be considered a responder for the primary efficacy endpoint. This is a much more challenging threshold than the achievement of a PGA-C score of clear or almost clear alone, when a patient need only change from mild (2) to almost clear (1) to be considered a responder. This more stringent criterion is used, as a change from the low end of the mild range to the high end of the almost clear range may not represent a clinically meaningful change.

The clinical significance of objective changes in disease severity were confirmed by the improvement in patientreported measures. Improvements in health-related quality of life, as indicated by DLQI, reflected the changes seen in PGA-C and PASI. Greater improvements in pruritus were seen compared with vehicle in both tofacitinib BID dosing groups from Day 2 of dosing and for $2 \%$ tofacitinib QD from Day 3. The improvements in pruritus from baseline were likely clinically meaningful (defined as a LSM decrease from baseline in
ISI of 2 points based on analyses conducted with oral tofacitinib therapy for psoriasis) [19] and were seen with $1 \%$ tofacitinib BID from Day 4 through Day 14, with $2 \%$ tofacitinib BID from Day 5 through Day 14, and with $2 \%$ tofacitinib QD from Day 8 through Day 14 (Fig. 3a).

Previous studies have shown oral tofacitinib improved patient-reported pruritus in moderate to severe psoriasis $[19,24,25]$. This is a direct effect, independent from improvements in clinician-reported signs of psoriasis severity [24], with a statistically significant improvement occurring as early as the second day of dosing [26]. Topical tofacitinib also improves pruritus in patients with atopic dermatitis [27].

A very rapid reduction in ISI was seen on initiation of treatment, with a significant reduction in pruritus with both $2 \%$ and $1 \%$ tofacitinib BID compared with vehicle BID as early as the day following the initial dose. Although pruritus is a common feature of psoriasis, the underlying pathogenesis is not understood. Impaired innervation and neuropeptide imbalance in psoriatic skin may be involved; other potential mechanisms include increased expression of IL-2, the opioid system, prostanoids, IL-31, serotonin, proteases and/or vascular abnormalities [28, 29]. Tofacitinib inhibition of JAK may suppress pruritus by blocking signaling via IL-31 [30, 31] and reducing expression of IL-2 [32]. As neuropeptides have a role in the pathogenesis of both psoriasis and pruritus, increased expression of substance $\mathrm{P}$ receptor, high-affinity nerve growth factor receptor or calcitonin gene-related peptide receptor may be involved [29].

Overall, topically administered tofacitinib had an acceptable safety profile, with no clinically meaningful differences in the incidence of AEs or SAEs between tofacitinib and vehicle treatment groups. The incidence of AEs coding to the MedDRA Infections and Infestations system organ class was higher in patients receiving vehicle than patients receiving tofacitinib. None of the 
side effects associated with topical application of potent corticosteroids were observed.

The range of observed plasma concentrations of tofacitinib from the PK analysis showed significant overlap between the dose strengths and regimens. Both $\mathrm{AUC}_{\text {tau }}$ and maximum observed plasma concentration were higher in patients with QD administration than with BID administration, which was not expected. In addition to dose strength and regimen, PK exposure is likely related to the treatment BSA and/or ointment application rate and this may be contributing to the lack of clear differentiation between the regimens.

In Phase 3 studies of oral tofacitinib in patients with moderate to severe psoriasis, serious infections and herpes zoster infections were associated with tofacitinib treatment $[14,15]$. Based on an exposure-response analysis of oral tofacitinib psoriasis data, an average tofacitinib exposure of $12.4 \mathrm{ng} / \mathrm{mL}$ was not associated with increased incidence rates of serious infections and herpes zoster infections when compared to patients treated with placebo (unpublished observations). In the current study, more than $83 \%$ of tofacitinib levels measured in plasma from patients in all active treatment groups were $<1.0 \mathrm{ng} / \mathrm{mL}$, which represents a $>12$-fold margin to the exposure levels for oral tofacitinib with no increased incidence rates for serious and herpes zoster infections relative to placebo observed in the oral tofacitinib Phase 3 psoriasis program.

\section{Study limitations}

To form the basis for further clinical development, this Phase $2 \mathrm{~b}$ estimation study used the 2 -sided $80 \%$ confidence interval as the pre-specified confidence level per study protocol, whereas in a Phase 3 trial the more rigorous $95 \%$ confidence interval or 0.05 significance level would be used. The stringent eligibility criteria of a Phase $2 \mathrm{~b}$ clinical study generally exclude some patients who may have been considered for topical treatment outside of the clinical trial environment. The numbers of patients included in the study ( 70 per treatment group) is a relatively small sample size. Caution is therefore needed in extrapolating findings to real-world clinical practice. No active comparator to tofacitinib was included so efficacy was not assessed relative to another agent with a known therapeutic effect in psoriasis. No formal statistical comparison was made between QD and BID application, as study sites were assigned to either QD or BID regimens, not both. As such, the dosing regimens essentially represent two separate sub-studies.

\section{Conclusions}

This small Phase 2b study demonstrated that topical treatment with the JAK inhibitor tofacitinib in an ointment formulation provided improvement in the clinical signs of psoriasis for patients with mild to moderate chronic plaque psoriasis. Based on the prespecified primary efficacy endpoint, which assesses clinical signs, tofacitinib as a $2 \%$ ointment formulation applied either QD or BID showed significantly greater efficacy compared with vehicle at Week 8 , but not at Week 12 , and not as a $1 \%$ ointment formulation. Acceptable safety and local tolerability profiles for both QD and BID dosing regimens were observed during 12 weeks of treatment.

\section{Additional files}

Additional file 1: Figure S1. Change from baseline in DLQI through Week 16. Table S1. Serious adverse events by treatment group. Table S2. Number and percent of plasma tofacitinib concentration samples (pre-dose and post-dose) by concentration range - available data mild/moderate - post hoc. Table S3. Summary of plasma tofacitinib pharmacokinetic parameters - available data at week 4 - mild/moderate post hoc. (DOCX $238 \mathrm{~kb}$ )

Additional file 2: List of independent ethics committees or institutional review boards. (DOCX $31 \mathrm{~kb}$ )

\section{Abbreviations}

AE: Adverse event; ALT: Alanine aminotransferase; AST: Aspartate aminotransferase; $A \cup C_{\text {tau: }}$ : Area under the plasma concentration time profile from time zero to the time tau; BID: Twice daily; BMl: Body mass index; BSA: Body surface area; Cl: Confidence interval; CPK: Creatine phosphokinase; DLQI: Dermatology Life Quality Index; IL: Interleukin; ISI: Itch Severity Item; JAK: Janus kinase; LSM: Least squares mean; MedDRA: Medical Dictionary for Regulatory Activities; PASI: Psoriasis Area and Severity Index; PASI75: Psoriasis Area and Severity Index 75; PGA-C: Calculated Physician's Global Assessment; PGA-Cm: Calculated Physician's Global Assessment of patients with mild to moderate plaque psoriasis at baseline; PK: Pharmacokinetic; QD: Once daily; SAE: Serious adverse event; SD: Standard deviation; SE: Standard error; ULN: Upper limit of normal

\section{Acknowledgments}

The authors would like to thank the investigators and staff who managed this clinical study, the patients who participated in the study, and Shahbaz Khan, MD, who provided clinical support.

This study was sponsored by Pfizer Inc. Medical writing and editorial support under the direction of the authors was provided by Carole Evans of Complete Medical Communications and funded by Pfizer Inc.

\section{Funding}

This study was sponsored by Pfizer Inc. Study design, data collection, analysis and interpretation of results were funded by Pfizer Inc. Pfizer employees participated in study design, data analysis and interpretation.

\section{Availability of data and materials}

The data supporting the conclusions of this article are included within the article and its additional files.

\section{Authors' contributions}

$K P, R B, M G, S R F, L I$, JS and ZD were all investigators on the study. All authors were involved in data interpretation; and manuscript drafting, reviewing and development. All authors read and approved the final manuscript.

\section{Competing interests}

KA Papp has been a consultant for AbbVie, Amgen, Anacor, Active Biotech, Allergan, Astellas, AstraZeneca, Basilea, Bayer, Baxter, Biogen-Idec, Boehringer Ingelheim, Celgene, Dermira, Eli Lilly, Forward Pharma, Galderma, Genentech, GSK, Incyte, Janssen, Kyowa Hakko Kirin, Kythera, Leo Pharma Inc., Medlmmune, Merck (MSD), Merck-Serono, Novartis, Pfizer Inc, Regeneron, Rigel, Roche, Takeda, UCB, Valeant, and Xenon; a speaker for AbbVie, Amgen, Allergan, Astellas, AstraZeneca, Basilea, Bayer, Baxter, Biogen-Idec, Boehringer 
Ingelheim, Celgene, Dermira, Eli Lilly, Galderma, Genentech, Janssen, Leo Pharma Inc., Medlmmune, Merck (MSD), Merck-Serono, Novartis, Pfizer Inc, Regeneron, UCB, and Valeant; and an investigator for AbbVie, Amgen, Anacor, Active Biotech, Allergan, Astellas, AstraZeneca, Basilea, Bayer, Baxter, Biogen-ldec, Boehringer Ingelheim, Celgene, Dermira, Eli Lilly, Forward Pharma, Galderma, Genentech, GSK, Incyte, Janssen, Kyowa Hakko Kirin, Kythera, Leo Pharma Inc., Medlmmune, Merck (MSD), Merck-Serono, Novartis, Pfizer Inc, Regeneron, Rigel, Roche, Takeda, UCB, and Valeant.

R Bissonnette has participated in advisory boards for AbbVie, Amgen, Janssen, and Merck. He has received consulting support and/or speaking support from AbbVie, Amgen, Celgene, Eli Lilly and Company, Galderma, Incyte, Janssen, Leo Pharma, Merck, and Novartis. He is an investigator for and his institution receives grant support from AbbVie, Amgen, Eli Lilly, GSKStiefel, Merck, Novartis, Pfizer Inc, Kineta, Incyte, Janssen, and Leo Pharma. M Gooderham has received research, speaking and/or consulting support from a variety of companies including AbbVie, Amgen, Boehringer Ingelheim, Celgene, Galderma, Janssen, Kyowa Hakko Kirin, Leo Pharma Inc Lilly, Medlmmune, Merck, Novartis, Pfizer Inc, Regeneron, and Roche. SR Feldman has received research, speaking and/or consulting support from a variety of companies including Galderma, GSK/Stiefel, Leo Pharma, Baxter, Boehringer Ingelheim, Mylan, Celgene, Pfizer Inc, Valeant, AbbVie, Cosmederm, Anacor, Astellas, Janssen, Lilly, Merck, Merz, Novartis, Qurient, National Biological Corporation, Caremark, Advance Medical, Suncare Research, Informa, UpToDate and National Psoriasis Foundation. He is founder and majority owner of www.DrScore.com, and founder and partowner of Causa Research, a company dedicated to enhancing patients' adherence to treatment. He has participated in advisory boards for AbbVie, Boehringer Ingelheim, Merck Novartis, and Pfizer Inc; has served as a consultant for AbbVie, Amgen, Celgene, Galderma Laboratories, Lilly, Mylan, Novartis, and Pfizer Inc, and is a member of speakers' bureaus for Celgene, Galderma, and Janssen.

$\mathrm{L}$ Iversen has served as a consultant and/or paid speaker for and/or participated in clinical trials sponsored by companies that manufacture drugs used for the treatment of psoriasis, including AbbVie, Almirall, Amgen, Celgene, Centocor, Eli Lilly, Janssen-Cilag, Leo Pharma, Merck (MSD), Novartis, and Pfizer Inc.

J Soung has received research, speaking and/or consulting support from a variety of companies including Janssen, Eli Lilly, Amgen, AbbVie, Merz, Pfizer Inc, Galderma, Valeant, National Psoriasis Foundation, Cassiopea, Celgene, Actavis, Actelion, and GSK.

Z Draelos received a financial grant from Pfizer Inc as an investigator to conduct the research described in this paper.

C Mamolo, V Purohit, C Wang, and W Ports are employees and shareholders of Pfizer Inc.

\section{Consent for publication}

Not applicable.

\section{Ethics approval and consent to participate}

The study was performed in compliance with the Declaration of Helsinki and the Good Clinical Practice Guidelines established by the International Council for Harmonisation. The study protocol was approved by the institutional review board at each investigational center (Additional file 2). All patients provided written, informed consent.

\section{Author details}

${ }^{1} \mathrm{~K}$ Papp Clinical Research and Probity Medical Research Inc, Waterloo, ON, Canada. ${ }^{2}$ Innovaderm Research, Montreal, QC, Canada. ${ }^{3}$ SKiN Centre for Dermatology and Probity Medical Research Inc, Peterborough, and Queens University, Kingston, ON, Canada. "Wake Forest Baptist Health, Winston-Salem, NC, USA. ${ }^{5}$ Aarhus University Hospital, Aarhus, Denmark. ${ }^{6}$ Southern California Dermatology, Santa Ana, CA, USA. ${ }^{7}$ Dermatology Consulting Services, High Point, NC, USA. ${ }^{8}$ Pfizer Worldwide Biopharmaceuticals, Global Innovative Pharma Business, Groton, CT, USA.

Received: 3 April 2016 Accepted: 9 September 2016

\section{Published online: 03 October 2016}

\section{References}

1. World Health Assembly 67. Psoriasis 2014. http://apps.who.int/gb/ebwha/ pdf_files/WHA67/A67_R9-en.pdf. Accessed 27 Jan 2016.
2. Stern RS, Nijsten T, Feldman SR, Margolis DJ, Rolstad T. Psoriasis is common, carries a substantial burden even when not extensive, and is associated with widespread treatment dissatisfaction. J Investig Dermatol Symp Proc. 2004:9:136-9.

3. Yeung H, Takeshita J, Mehta NN, Kimmel SE, Ogdie A, Margolis DJ, et al. Psoriasis severity and the prevalence of major medical comorbidity: a population-based study. JAMA Dermatol. 2013;149:1173-9.

4. van de Kerkhof PC, Reich K, Kavanaugh A, Bachelez H, Barker J, Girolomoni G, et al. Physician perspectives in the management of psoriasis and psoriatic arthritis: results from the population-based multinational assessment of psoriasis and psoriatic arthritis survey. J Eur Acad Dermatol Venereol. 2015;29:2002-10.

5. Menter A, Korman NJ, Elmets CA, Feldman SR, Gelfand JM, Gordon KB, et al. Guidelines of care for the management of psoriasis and psoriatic arthritis. Section 3. Guidelines of care for the management and treatment of psoriasis with topical therapies. J Am Acad Dermatol. 2009;60:643-59.

6. Papp K, Gulliver W, Lynde C, Poulin Y, Ashkenas J. Canadian guidelines for the management of plaque psoriasis: overview. J Cutan Med Surg. 2011:15:210-9.

7. National Clinical Guideline Centre (UK). Psoriasis: Assessment and Management of Psoriasis. London: Royal College of Physicians (UK); 2012 Oct. National Institute for Health and Clinical Excellence: Guidance. 2012. https://www.nice.org.uk/guidance/cg153. Accessed 27 Jan 2016.

8. van de Kerkhof PC, Kragballe K, Segaert S, Lebwohl M. Factors impacting the combination of topical corticosteroid therapies for psoriasis: perspectives from the international psoriasis council. J Eur Acad Dermatol Venereol. 2011;25:1130-9.

9. Brodell RT, Bruce S, Hudson CP, Weiss JS, Colon LE, Johnson LA, et al. A multi-center, open-label study to evaluate the safety and efficacy of a sequential treatment regimen of clobetasol propionate $0.05 \%$ spray followed by Calcitriol $3 \mathrm{mg} / \mathrm{g}$ ointment in the management of plaque psoriasis. J Drugs Dermatol. 2011;10:158-64.

10. Barnes L, Altmeyer P, Forstrom L, Stenstrom MH. Long-term treatment of psoriasis with calcipotriol scalp solution and cream. Eur J Dermatol. 2000;10:199-204.

11. Mason AR, Mason J, Cork M, Dooley G, Edwards G. Topical treatments for chronic plaque psoriasis. Cochrane Database Syst Rev. 2009:CD005028.

12. Menter A, Korman NJ, Elmets CA, Feldman SR, Gelfand JM, Gordon KB, et al. Guidelines of care for the management of psoriasis and psoriatic arthritis: section 6. Guidelines of care for the treatment of psoriasis and psoriatic arthritis: case-based presentations and evidence-based conclusions. J Am Acad Dermatol. 2011;65:137-74.

13. Armstrong AW, Robertson AD, Wu J, Schupp C, Lebwohl MG. Undertreatment, treatment trends, and treatment dissatisfaction among patients with psoriasis and psoriatic arthritis in the United States: findings from the national psoriasis foundation surveys, 2003-2011. JAMA Dermatol. 2013;149:1180-5.

14. Papp KA, Menter MA, Abe M, Elewski B, Feldman SR, Gottlieb AB, et al. Tofacitinib, an oral Janus kinase inhibitor, for the treatment of chronic plaque psoriasis: results from two, randomised, placebo-controlled, Phase 3 trials. Br J Dermatol. 2015;173:949-61.

15. Bissonnette $R$, Iversen $L$, Sofen $H$, Griffiths CEM, Foley $P$, Romiti $R$, et al. Tofacitinib withdrawal and retreatment in moderate-to-severe chronic plaque psoriasis: a randomized controlled trial. $\mathrm{Br} J$ Dermatol. 2015;172:1395-406.

16. Bachelez $\mathrm{H}$, van de Kerkhof PCM, Strohal R, Kubanov A, Valenzuela F, Lee JH, et al. Tofacitinib versus etanercept or placebo in moderate-to-severe chronic plaque psoriasis: a phase 3 randomised non-inferiority trial. Lancet. 2015;386:552-61.

17. Ports WC, Khan S, Lan S, Lamba M, Bolduc C, Bissonnette R, et al. A randomized phase $2 a$ efficacy and safety trial of the topical Janus kinase inhibitor tofacitinib in the treatment of chronic plaque psoriasis. Br J Dermatol. 2013;169:137-45.

18. Cappelleri JC, Bushmakin AG, Harness J, Mamolo C. Psychometric validation of the physician global assessment scale for assessing severity of psoriasis disease activity. Qual Life Res. 2013;22:2489-99.

19. Mamolo CM, Bushmakin AG, Capelleri JC. Application of the Itch Severity Score in patients with moderate-to-severe plaque psoriasis: clinically important difference and responder analyses. J Dermatolog Treat. 2015;26:121-3.

20. Cochran WG. Some methods of strengthening the common $X 2$ tests. Biometrics. 1954;10:417-51. 
21. Mantel N, Haenszel W. Statistical aspects of the analysis of data from retrospective studies of disease. J Natl Cancer Inst. 1959;22:719-48.

22. SAS Institute Inc. SAS/STAT 9.3 User's Guide. Cary, North Carolina: SAS Institute Inc; 2011

23. Krueger J, Clark JD, Suárez-Fariñas M, Fuentes-Duculan J, Cueto I, Wang CQ, Tan H, Wolk R, Rottinghaus ST, Whitley MZ, Valdez H, von Schack D, O'Neil SP, Reddy PS, Tatulych S, for the A3921 147 Study Investigators. Tofacitinib attenuates pathologic immune pathways in psoriasis: a randomized Phase 2 study. J Allergy Clin Immunol. 2016;137:1079-90.

24. Bushmakin AG, Mamolo C, Cappelleri JC, Stewart M. The relationship between pruritus and the clinical signs of psoriasis in patients receiving tofacitinib. J Dermatolog Treat. 2015;26:19-22.

25. Luger T, Cappelleri J, Bushmakin A, Mallbris L, Mamolo C. Clinically meaningful improvement in pruritus with tofacitinib: results from a phase 3 program [abstract]. In: The 23rd World Congress of Dermatology Abstracts and Proceedings Vancouver. 2015. http://derm2015.org/program/abstractvolume/. Accessed 27 Jan 2016.

26. Mamolo C, Harness J, Tan H, Menter A. Tofacitinib (CP-690,550), an oral Janus kinase inhibitor, improves patient-reported outcomes in a phase $2 \mathrm{~b}$, randomized, double-blind, placebo-controlled study in patients with moderate-to-severe psoriasis. J Eur Acad Dermatol Venereol. 2014;28:192-203.

27. Bissonnette R, Papp KA, Poulin Y, Gooderham M, Raman M, Mallbris L, Wang C, Purohit Y, Mamolo C, Papacharalambous J, Ports WC. Topical tofacitinib for atopic dermatitis: A Phase 2a randomised trial. Br J Dermatol. 2016. Epub ahead of print.

28. Chang SE, Han SS, Jung HJ, Choi JH. Neuropeptides and their receptors in psoriatic skin in relation to pruritus. Br J Dermatol. 2007;156:1272-7.

29. Reich A, Szepietowski JC. Mediators of pruritus in psoriasis. Mediators Inflamm. 2007;2007:64727.

30. Cornelissen C, Luscher-Firzlaff J, Baron JM, Luscher B. Signaling by IL-31 and functional consequences. Eur J Cell Biol. 2012;91:552-66.

31. Sonkoly E, Muller A, Lauerma Al, et al. IL-31: a new link between T cells and pruritus in atopic skin inflammation. J Allergy Clin Immunol. 2006;117:411-7.

32. Flanagan ME, Blumenkopf TA, Brissette WH, Brown MF, Casavant JM, Shang-Poa S, et al. Discovery of CP-690,550: a potent and selective Janus Kinase (JAK) inhibitor for the treatment of autoimmune diseases and organ transplant rejection. J Med Chem. 2010;53:8468-84.

\section{Submit your next manuscript to BioMed Central and we will help you at every step:}

- We accept pre-submission inquiries

- Our selector tool helps you to find the most relevant journal

- We provide round the clock customer support

- Convenient online submission

- Thorough peer review

- Inclusion in PubMed and all major indexing services

- Maximum visibility for your research

Submit your manuscript at www.biomedcentral.com/submit

) Biomed Central 